\title{
Sign of the crossed conductances at a FSF double interface
}

\author{
R. Mélin* \\ Centre de Recherches sur les Très Basses Températures (CRTBT $\left.T^{\dagger}\right)$, \\ CNRS, BP 166, 38042 Grenoble Cedex 9, France \\ D. Feinberg \\ Laboratoire d'Etudes des Propriétés Electroniques des Solides (LEPES $\left.S^{\ddagger}\right)$, \\ CNRS, BP 166, 38042 Grenoble Cedex 9, France
}

\begin{abstract}
Crossed conductance in hybrid Ferromagnet / Superconductor / Ferromagnet (FSF) structures results from the competition between normal transmission and Andreev reflection channels. Crossed Andreev reflection (CAR) and elastic cotunneling (EC) between the ferromagnets are dressed by local Andreev reflections, which play an important role for transparent enough interfaces and intermediate spin polarizations. This modifies the simple result previously obtained at lowest order, and can explain the sign of the crossed resistances in a recent experiment [D. Beckmann et al., cond-mat/0404360. This holds both in the multiterminal hybrid structure model (where phase averaging over the Fermi oscillations is introduced "by hand" within the approximation of a single non local process) and for infinite planar interfaces (where phase averaging naturally results in the microscopic solution with multiple non local processes).

PACS numbers: $74.50 .+\mathrm{r}, 74.78 . \mathrm{Na}, 74.78 . \mathrm{Fk}$
\end{abstract}

\section{INTRODUCTION}

Andreev reflection ${ }^{1.2}$ is the mechanism by which charge is transported at normal metal / superconductor (NS) interfaces at voltages below the superconducting gap. A spin-up electron from the $\mathrm{N}$ electrode is reflected as a spin-down hole while a Cooper pair is transfered in the superconductor. Multiterminal geometries are of particular interest. For instance the sign of the crossed conductance between two electrodes connected to a same superconductor can be either positive or negative depending on the relative strengths of the "normal" (electron-electron) and "anomalous" (electron-hole) channels $3,4,5$. Multiprobe structures are especially interesting when the electrodes are ferromagnets. Actually, Andreev reflection also exists in ferromagnet / superconductor (FS) junctions but is suppressed as the spin polarization of the ferromagnet increases 6.7.8. It was shown 9.10 .11 .12 .13 .14 .15 .16 .17 .19 .20 .21 that "crossed" Andreev reflections (CAR) between two ferromagnetic electrodes, where spin-up electron and outgoing spin-down hole belong to different electrodes, give rise to interesting magnetoresistance effects. For instance, the latter process is favored by antiparallel spin polarizations. The fundamental problem of calculating the scattering matrix $S_{i j}$ of a NSN structure has been solved in some limits. The authors of Ref. 20 use a "fork" geometry where the two electrodes merge at the superconductor. In con-

*melin@grenoble.cnrs.fr

†U.P.R. 5001 du CNRS, Laboratoire conventionné avec l'Université Joseph Fourier

${ }^{\ddagger}$ U.P.R. 11 du CNRS, Laboratoire conventionné avec l’Université Joseph Fourier trast, in Refs. 9.10 (like in a recent experiment ${ }^{21}$ ), two distinct $\mathrm{F} / \mathrm{S}$ contacts (denoted here as $a, b$ ) are separated by a distance $R$. Yamashita et al. ${ }^{22}$ used a generalization of the BTK approach in this geometry ${ }^{23}$. As a common result, CAR is possible if $R<\xi$, the superconducting coherence length, but is also reduced by an algebraic factor. Andreev reflection becomes in this geometry a genuine mesoscopic effect, non-local at the scale of the physical contacts. On the other hand, in the normal channel, transmission between the two electrodes is also possible. Since it is spin-conserving, it is favored by parallel spin polarizations. In a tunnel model for the two $\mathrm{F} / \mathrm{S}$ junctions, CAR and EC involve the virtual creation and propagation of a quasiparticle in the superconductor. For the normal channel, this is similar to cotunneling, introduced in the context of transmission across a Coulomb blockaded quantum dot ${ }^{24}$. The superconducting gap replacing the Coulomb energy, and the normal crossed process has been called "elastic cotunneling" in Ref. 10.

Other manifestations of spatially separated pair correlations were obtained in the study of equilibrium properties of FSF trilayers 11.25.26.27.28.29. It was shown 11.25 .26 within a model of multiterminal hybrid structure that the self-consistent superconducting gap can be larger in the parallel alignment. The same result was obtained for the FSF trilayer with atomic thickness, for half-metal ferromagnets 27 and Stoner ferromagnets 28 . However simulations with a finite thickness ${ }^{28.29}$ showed that pairbreaking dominates for strong ferromagnets as the thickness of the superconductor is larger than the Fermi wavelength (with therefore the superconducting gap larger in the antiparallel alignment).

The goal of the theory of crossed conductances at a FSF double interface is to calculate the conductances of a FSF structure for any relative alignments of the spin 
polarizations. In a first approximation we neglect the dependence of the superconducting gap on the relative spin orientation in the ferromagnets, which is a consistent assumption if the size of the contacts is much smaller than the superconducting coherence length ${ }^{30}$. The first approach to this problem was through Landauer formalism ${ }^{9}$. Lowest order perturbation theory $\underline{10}$ gives an interpretation in terms of CAR and EC processes. The effect of non collinear ferromagnets was also investigated ${ }^{13.14}$, with the aim of describing transport of Cooper pairs at the interface between a superconductor and a ferromagnet containing a domain wall. The Josephson effect between two superconductors connected by two spatially separated conduction channels was also examined 13 and it was found that there is no Josephson effect within lowest order perturbation theory unless the length of the ferromagnets is smaller than the elastic mean free path, a condition that is not usually verified in experiments. Toy models for more complicated geometries involving Aharonov-Bohm effects related to crossed correlations were also investigated ${ }^{15}$. Disorder effects were also discussed recently for tunnel interfaces ${ }^{14.16}$ and it was found that the geometrical reduction is less severe in the presence of disorder in the superconductor (dirty limit). Another geometry with a normal metal island connected to one superconductor and two ferromagnets was proposed in Ref. 17. Noise correlations were also discussed $^{18}$ and recently within lowest order perturbation theory $\stackrel{19}{ }$.

CAR and EC were probed in a recent experiment by Beckmann et $a l^{21}$. Driving a current through one contact induces a voltage in the other one. The overall experimental results (the non-local resistance) are in agreement with theory except for the sign of the effect: it was predicted theoretically 10.20 that for tunnel barriers the induced currents in the parallel and antiparallel spin orientations have an opposite sign whereas in experiments ${ }^{21}$ they have the same sign. Here we resolve this apparent contradiction by noting that the large interface transparencies used in experiments imply that the CAR and EC processes are "dressed" by local Andreev reflections at the two FS interfaces. This dressing is easily transcripted at zero temperature in terms of a perturbative expansion for the Keldysh Green's functions. Due to the strong damping of quasiparticles in the superconductor, a reasonable approximation involves a single non local propagator while local ones are treated to all orders. We also carry out numerical simulations of infinite planar interfaces in which multiple non local processes are taken into account and find a good agreement with the analytical approach.

The article is organized as follows. Preliminaries are given in section [II The properties of multiterminal hybrid structures are investigated in section III] Infinite planar interfaces are investigated in section [IV] Concluding remarks are given in section $\nabla$

\section{PRELIMINARIES}

In this section we provide the form of the Green's functions and Hamiltonians that we use throughout the article.

\section{A. Hamiltonians}

The superconductor is described by the BCS Hamiltonian ${ }^{31}$ :

$$
\begin{aligned}
\mathcal{H}_{\mathrm{BCS}} & =\sum_{\mathbf{k}, \sigma} \epsilon(k) c_{\mathbf{k}, \sigma}^{+} c_{\mathbf{k}, \sigma} \\
& +\Delta \sum_{\mathbf{k}}\left(c_{\mathbf{k}, \uparrow}^{+} c_{\mathbf{k}, \downarrow}^{+}+c_{\mathbf{k}, \downarrow} c_{\mathbf{k}, \uparrow}\right),
\end{aligned}
$$

where $\epsilon(k)=\hbar^{2} k^{2} / 2 m$ is the free electron dispersion relation and $\Delta$ the superconducting gap. The ferromagnets are described by the Stoner model

$$
\begin{aligned}
\mathcal{H}_{\text {Stoner }} & =\sum_{\mathbf{k}, \sigma} \epsilon(k) c_{\mathbf{k}, \sigma}^{+} c_{\mathbf{k}, \sigma} \\
& -h_{\mathrm{ex}} \sum_{\mathbf{k}}\left(c_{\mathbf{k}, \uparrow}^{+} c_{\mathbf{k}, \uparrow}-c_{\mathbf{k}, \downarrow}^{+} c_{\mathbf{k}, \downarrow}\right),
\end{aligned}
$$

where $h_{\mathrm{ex}}$ is the exchange field.

\section{B. Green's functions in reciprocal space}

For collinear magnetizations the Nambu representation corresponds to two $2 \times 2$ matrices, one in the sector $S_{z}=1 / 2$ and the other in the sector $S_{z}=-1 / 2\left(S_{z}\right.$ is the projection of the spin along the quantization axis chosen parallel to the exchange field). The advanced Green's function in the sector $S_{z}=1 / 2$ is given at zero temperature by

$$
\begin{aligned}
& \hat{g}_{\mathbf{x}, \mathbf{y}}^{A}\left(t, t^{\prime}\right)=-i \theta\left(t-t^{\prime}\right) \\
& \left(\begin{array}{ll}
\left\langle\left\{c_{\mathbf{x}, \uparrow}(t), c_{\mathbf{y}, \uparrow}^{+}\left(t^{\prime}\right)\right\}\right\rangle & \left\langle\left\{c_{\mathbf{x}, \uparrow}(t), c_{\mathbf{y}, \downarrow}\left(t^{\prime}\right)\right\}\right\rangle \\
\left\langle\left\{c_{\mathbf{x}, \downarrow}^{+}(t), c_{\mathbf{y}, \uparrow}^{+}\left(t^{\prime}\right)\right\}\right\rangle & \left\langle\left\{c_{\mathbf{x}, \downarrow}^{+}(t), c_{\mathbf{y}, \downarrow}\left(t^{\prime}\right)\right\}\right\rangle
\end{array}\right),
\end{aligned}
$$

where $\mathbf{x}$ and $\mathbf{y}$ are two arbitrary sites and $\{.,$.$\} is an$ anticommutator. Using the Hamiltonian (10) one obtains the advanced Green's function in reciprocal space ${ }^{32}$ :

$$
\begin{aligned}
g^{1,1, A}(\xi, \omega)= & \frac{u_{k}^{2}}{\left(\omega-\mu_{S}\right)-E_{k}-i \eta_{S}} \\
+ & \frac{v_{k}^{2}}{\left(\omega-\mu_{S}\right)+E_{k}-i \eta_{S}} \\
f^{1,2, A}(\xi, \omega)= & -\Delta \frac{1}{\left(\omega-\mu_{S}\right)-E_{k}-i \eta_{S}} \\
& \times \frac{1}{\left(\omega-\mu_{S}\right)+E_{k}-i \eta_{S}},
\end{aligned}
$$




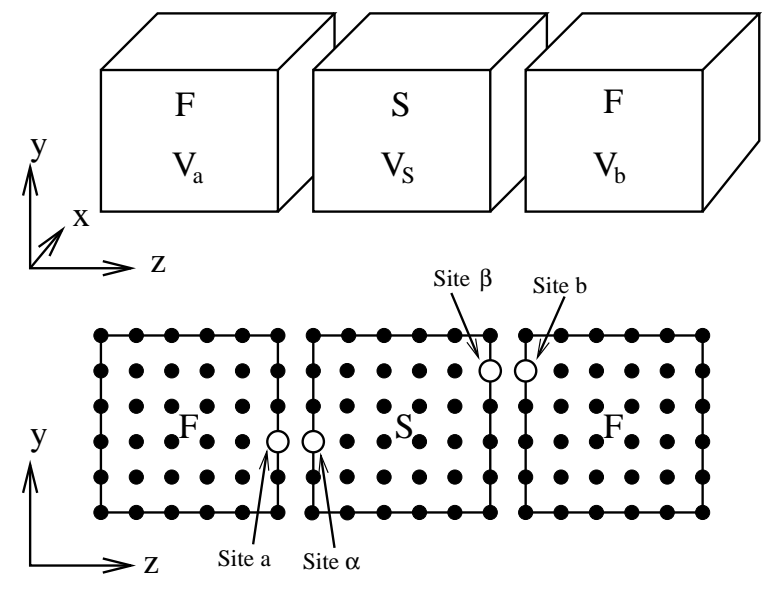

(a)

FIG. 1: (a) Schematic representation of the Andreev reflection geometry in a FSF structure. (b) Schematic representation of the tight-binding Hamiltonian cubic lattice and notation for the sites $a, \alpha, \beta$ and $b$. (b) corresponds to a cut in the $(y, z)$ plane. Voltages $V_{a}$ and $V_{b}$ are applied on the two ferromagnets and a voltage $V_{S}$ is applied on the superconductor.

where $\mu_{S}$ is the chemical potential in the superconductor, $\xi=\hbar^{2} k^{2} / 2 m-\mu_{S}$ is the kinetic energy with respect to the Fermi level, $E_{k}=\sqrt{\Delta^{2}+\xi_{k}^{2}}$ is the quasiparticle energy, $u_{k}^{2}=\left(1+\xi_{k} / E\right) / 2$ and $v_{k}^{2}=\left(1-\xi_{k} / E\right) / 2$ are the BCS coherence factors, and $\eta_{S}$ is a small parameter related to inelastic processes in the superconductor (a typical ${ }^{33.34}$ value in experiments is $\eta_{S}=10^{-2} \Delta$ ).

The "11" component of the Green's function of a ferromagnet is given by

$$
g_{a, a}^{1,1}(\xi, \omega)=\frac{1}{\left(\omega-\mu_{a}\right)-\xi+h_{\mathrm{ex}}-i \eta_{F}},
$$

where $\mu_{a}$ is the chemical potential in the ferromagnet "a". A similar expression is obtained for $g_{a, a}^{2,2}$. The parameter $\eta_{F}$ contains information about decoherence in the ferromagnet ${ }^{35}$.

\section{Green's functions in real space}

It is useful in transport theory to manipulate Green's functions parameterized by spatial variables. To carry out the Fourier transform we define a short-scale cut-off corresponding to the lattice spacing $a_{0}$ of a cubic lattice tight-binding model where electrons hop between neighboring discrete sites like in Ref. 36 (see Fig. 1). The tight-binding and continuous models are equivalent since we consider low energies compared to the band-width.

The local Green's functions of a superconductor is defined as

$$
\hat{g}_{\alpha, \alpha}(\omega)=\int \frac{d^{3} \mathbf{k}}{(2 \pi)^{3}} \hat{g}(\xi, \omega)
$$

where $\alpha$ is a site in the superconductor (see Fig. 1), and where $\hat{g}(\xi, \omega)$ is given by Eqs. (4) and (5). Evaluating the integral over wave vector leads to

$$
\begin{aligned}
& \hat{g}_{\alpha, \alpha}(\omega)=\hat{g}_{\beta, \beta}(\omega) \\
= & \frac{\pi \rho_{S}}{\sqrt{\Delta^{2}-\left(\omega-\mu_{S}\right)^{2}}}\left[\begin{array}{cc}
-\left(\omega-\mu_{S}\right) & \Delta \\
\Delta & -\left(\omega-\mu_{S}\right)
\end{array}\right],
\end{aligned}
$$

where $\rho_{S}$ is the normal state density of state, $\omega$ is the energy and $\Delta$ is the superconducting gap. The Green's functions of the superconductor take the form ${ }^{37}$

$$
\begin{aligned}
& \hat{g}_{\alpha, \beta}(\omega)=\frac{\pi \rho_{S}}{k_{F} R} \exp \left(-\frac{R}{\xi(\omega)}\right) \\
& \left\{\frac{\sin \left(k_{F} R\right)}{\sqrt{\Delta^{2}-\left(\omega-\mu_{S}\right)^{2}}}\left[\begin{array}{cc}
-\left(\omega-\mu_{S}\right) & \Delta \\
\Delta & -\left(\omega-\mu_{S}\right)
\end{array}\right]\right. \\
& \left.+\cos \left(k_{F} R\right)\left[\begin{array}{cc}
-1 & 0 \\
0 & 1
\end{array}\right]\right\},
\end{aligned}
$$

where $\alpha$ and $\beta$ are two sites in the superconductor (see Fig. 1). $\xi(\omega)=\hbar v_{F} / \sqrt{\Delta^{2}-\left(\omega-\mu_{S}\right)^{2}}$ is the BCS coherence length. The local Green's functions of the ferromagnet is given by

$$
\hat{g}_{a, a}=i \pi\left[\begin{array}{cc}
\rho_{a, \uparrow} & 0 \\
0 & \rho_{a, \downarrow}
\end{array}\right],
$$

where $\rho_{a, \uparrow}$ and $\rho_{a, \downarrow}$ correspond to the spin-up and spindown density of states in the ferromagnet "a". A spin polarization $P$ corresponds to $\rho_{a, \uparrow}=\rho_{F}\left(1+P_{a}\right)$ and $\rho_{a, \downarrow}=\rho_{F}\left(1-P_{a}\right)$. We discard the energy dependence in (10) since we are interested in energies of order $\Delta$, much smaller than the exchange field $h_{\mathrm{ex}}$ related to the spin polarization by $P \simeq h_{\mathrm{ex}} / \epsilon_{F}$, for $h_{\mathrm{ex}}$ small compared to $\epsilon_{F}$.

\section{Mixed Green's functions}

To describe infinite planar contacts we use Green's functions parameterized by the distance $R$ along the $z$ axis and the two wave-vectors $k_{x}$ and $k_{y}$. This parameterization is well suited for a situation where translation invariance holds in the $(x, y)$ plane parallel to the interface but not in the $z$ direction perpendicular to the interface. The kinetic energy $\xi$ is separated into the sum of $\xi_{\|}=\hbar^{2}\left(k_{x}^{2}+k_{y}^{2}\right) / 2 m-\mu_{S}$ and $\xi_{\perp}=\hbar^{2} k_{z}^{2} / 2 m$. The mixed Green's function is defined by

$$
\hat{g}\left(R, \xi_{\|}, \omega\right)=\int \frac{d k_{z}}{2 \pi} e^{i k_{z} R} \hat{g}(\xi, \omega),
$$

where $\hat{g}(\xi, \omega)$ is defined by Eqs. (4) and (5). After evaluating the Fourier transform with respect to $k_{z}$ we obtain

$$
\begin{aligned}
& \hat{g}^{A}\left(R, \xi_{\|}, \omega\right)=\frac{\sqrt{2 m a_{0}^{2}}}{2 \hbar} \exp \left(i k_{z} R\right) \\
& \times \frac{1}{\sqrt{i \sqrt{\Delta^{2}-\left(\omega-\mu_{S}-i \eta_{S}\right)^{2}}-\xi_{\|}}}
\end{aligned}
$$




$$
\begin{aligned}
& \left\{\frac{1}{\sqrt{\Delta^{2}-\left(\omega-\mu_{S}-i \eta_{S}\right)^{2}}}\right. \\
& \left.\times\left[\begin{array}{cc}
\omega-\mu_{S}-i \eta_{S} & -\Delta \\
-\Delta & \omega-\mu_{S}-i \eta_{S}
\end{array}\right]+i\left[\begin{array}{cc}
1 & 0 \\
0 & -1
\end{array}\right]\right\},
\end{aligned}
$$

with

$$
k_{z}=\frac{\sqrt{2 m}}{\hbar} \sqrt{i \sqrt{\left(\omega-\mu_{S}-i \eta_{S}\right)^{2}-\Delta^{2}}-\xi_{\|}} .
$$
by

The mixed Green's function of a ferromagnet is given

$$
\begin{aligned}
g_{1,1}^{A}\left(R, \xi_{\|}, \omega\right)= & -\frac{\sqrt{2 m a_{0}^{2}}}{\hbar} \exp \left(i k_{z} R\right) \\
& \times \frac{1}{\sqrt{-\left(\omega-\mu_{a}\right)+i \eta_{F}+\xi_{\|}-h}},
\end{aligned}
$$

with

$$
k_{z}=\frac{\sqrt{2 m}}{\hbar} \sqrt{\omega-\mu_{a}-i \eta_{F}+h_{\mathrm{ex}}} .
$$

\section{E. Transport properties}

The Green's functions $\hat{G}_{i, j}$ of the connected system are obtained by solving the Dyson equation. In a compact notation the Dyson equation takes the form $\hat{G}=\hat{g}+$ $\hat{g} \otimes \hat{\Sigma} \otimes \hat{G}$, where $\hat{\Sigma}$ is the self-energy corresponding to the tunnel Hamiltonian, $\otimes$ is a summation over spatial variables and a convolution over time variables, and $\hat{g}$ is the Green's function of the disconnected system with $\hat{\Sigma}=0$.

Tranport properties ${ }^{34.36}$ are obtained by evaluating the Keldysh Green's function

$$
\hat{G}^{+,-}=\left[\hat{I}+\hat{G}^{R} \otimes \hat{\Sigma}\right] \otimes \hat{g}^{+,-} \otimes\left[\hat{I}+\hat{\Sigma} \otimes \hat{G}^{A}\right] .
$$

The spin-up current through the link $a-\alpha$ is given by

$$
\begin{aligned}
I_{a, \alpha} & =\frac{e}{2 h} \int d \omega \operatorname{Tr}\left\{\left[\hat{t}_{a, \alpha} \hat{G}_{\alpha, a}^{+,-}(\omega)-\hat{t}_{\alpha, a} \hat{G}_{a, \alpha}^{+,-}(\omega)\right] \hat{\sigma}^{z}\right\} \\
& +\left(h_{\mathrm{ex}} \rightarrow-h_{\mathrm{ex}}\right),
\end{aligned}
$$

where the trace is a summation over the " 11 " and " 22 " components of the Green's function in the Nambu representation, and $\hat{\sigma}^{z}$ is one of the Pauli matrices. The term $\left(h_{\mathrm{ex}} \rightarrow-h_{\mathrm{ex}}\right)$ corresponds to a summation over the " 33 " and " 44 " components in the $4 \times 4$ Nambu representation 13 in the sector $S_{z}=-1 / 2$. For a normal metal the four components of the current are equal and we recover the usual form of the transport formula ${ }^{34.36}$.

\section{MULTITERMINAL HYBRID STRUCTURES}

\section{A. Transport formula}

We start with the geometry on Fig. 1 In the Hamiltonian approach, the exact geometry is unimportant : the results are the same for two lateral contacts (like in the experiment ${ }^{21}$ ) or opposite contacts (Fig. 11). Moreover we suppose in this section that the distance between the contacts is large compared to their transverse dimension so that the distance between sites $\alpha$ and $\beta$ (see Fig. (1) can be considered as approximately independent on the choice of the sites $\alpha$ and $\beta$ at the interfaces. This hypothesis does not hold in the infinite planar geometry (see section [V]). The total current flowing between the two electrodes is the sum of two contributions : i) the CAR current (Andreev process) circulating from both electrodes "a" and "b", and ii) the EC current flowing from "a" to "b". Those are given by

$$
\begin{aligned}
& I_{\mathrm{CAR}}\left(V_{a}, V_{b}\right)=2 \pi^{2} t_{a}^{2} t_{b}^{2} \frac{e}{h} \rho_{a, \uparrow} \rho_{b, \downarrow} \\
& \times\left\{\left[\int_{-e V_{b}}^{e V_{a}}+\int_{-e V_{a}}^{e V_{b}}\right] d \omega \overline{G_{\alpha, \beta}^{1,2, A}(\omega) G_{\beta, \alpha}^{2,1, R}(\omega)}\right\} \\
& +2 \pi^{2} t_{a}^{2} t_{b}^{2} \frac{e}{h} \rho_{a, \downarrow} \rho_{b, \uparrow} \\
& \times\left\{\left[\int_{-e V_{b}}^{e V_{a}}+\int_{-e V_{a}}^{e V_{b}}\right] d \omega \overline{G_{\alpha, \beta}^{2,1, A}(\omega) G_{\beta, \alpha}^{1,2, R}(\omega)}\right\},
\end{aligned}
$$

and

$$
\begin{aligned}
& I_{\mathrm{EC}}\left(V_{a}, V_{b}\right)=2 \pi^{2} t_{a}^{2} t_{b}^{2} \frac{e}{h} \rho_{a, \uparrow} \rho_{b, \uparrow} \\
& \times\left\{\left[\int_{e V_{b}}^{e V_{a}}+\int_{-e V_{a}}^{-e V_{b}}\right] d \omega \overline{G_{\alpha, \beta}^{1,1, A}(\omega) G_{\beta, \alpha}^{1,1, R}(\omega)}\right. \\
& +2 \pi^{2} t_{a}^{2} t_{b}^{2} \frac{e}{h} \rho_{a, \downarrow} \rho_{b, \downarrow} \\
& \times\left\{\left[\int_{e V_{b}}^{e V_{a}}+\int_{-e V_{a}}^{-e V_{b}}\right] d \omega \overline{G_{\alpha, \beta}^{2,2, A}(\omega) G_{\beta, \alpha}^{2,2, R}(\omega)}\right\} .
\end{aligned}
$$

Eq. (17) is valid for all values of the voltages $V_{a}, V_{b}$ and $V_{S}$. Eqs. (18) and (19) are valid for all values of $V_{a}$ and $V_{b}$ and we supposed that $V_{S}=0$ since one reference voltage can be chosen equal to zero.

The crossed conductance is defined ${ }^{4}$ as $\mathcal{G}_{a b}\left(V_{a}, V_{b}\right)=$ $\partial I_{a, \alpha}\left(V_{a}, V_{b}\right) / \partial V_{b}$, where $I_{a, \alpha}\left(V_{a}, V_{b}\right)=I_{\mathrm{CAR}}\left(V_{a}, V_{b}\right)+$ $I_{\mathrm{EC}}\left(V_{a}, V_{b}\right)$ is the total crossed current through the link $\alpha-a$. In the following we derive the currents (18) and (19) with respect to the voltage $V_{b}$. One contribution to the derivative is due to the upper and lower bounds in the integrals. A second contribution is due to the dependence of the Green's functions on $V_{b}$. However we deduce from Eq. (6) that $\hat{g}_{b, b}$ is independent on $V_{b}$ because the chemical potential is subtracted both in the energy $\omega-\mu_{b}$ and in the kinetic energy $\xi$. This second contribution to the derivative is thus vanishingly small. The crossed conductance can be expressed in terms of the spin polarizations $P_{a}$ and $P_{b}$ :

$$
\begin{aligned}
\mathcal{G}_{a b}= & 4 \pi^{2} t_{a}^{2} t_{b}^{2} \frac{e}{h} \rho_{F a} \rho_{F b}\{ \\
& \left(1-P_{a} P_{b}\right) \overline{G_{\alpha, \beta}^{1,2, A}\left(e V_{b}\right) G_{\beta, \alpha}^{2,1, R}\left(e V_{b}\right)} \\
& -\left(1+P_{a} P_{b}\right) \overline{G_{\alpha, \beta}^{1,1, A}\left(e V_{b}\right) G_{\beta, \alpha}^{1,1, R}\left(e V_{b}\right)}
\end{aligned}
$$



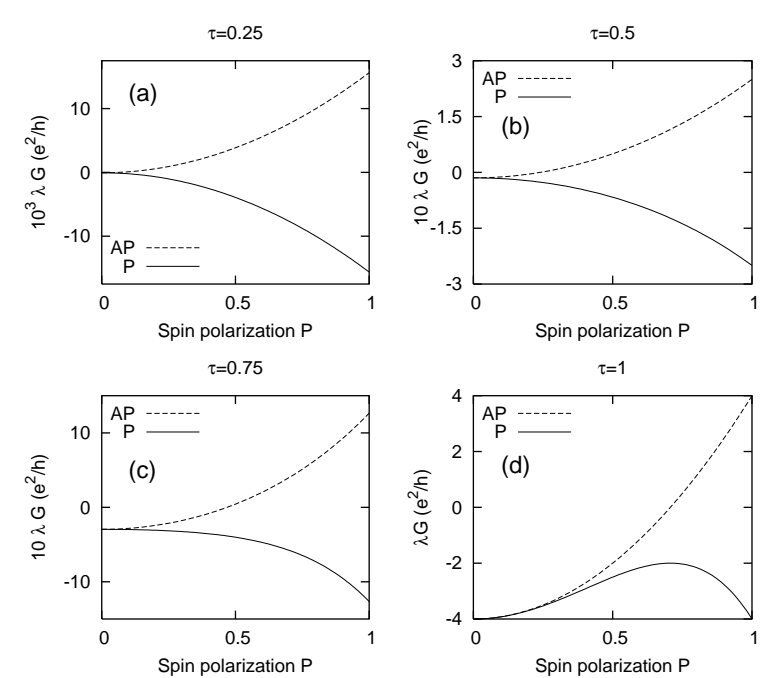

FIG. 2: Variation of the normalized linear crossed conductances $\lambda \mathcal{G}_{\mathrm{P}}(P)$ (solid line) and $\lambda \mathcal{G}_{\mathrm{AP}}(P)$ (dashed line) as a function of the spin polarization $P$, for $\tau=0.25$ (a), $\tau=0.5$ (b), $\tau=0.75$ (c), $\tau=2$ (d). Note the different scaling factors on the conductance axis. and $\tau=1$

$$
\left.+\left(e V_{b} \rightarrow-e V_{b}\right)\right\}
$$

Averaging over the Fermi oscillations $\frac{10.12}{12}$ is used to simulate an extended contact as the sum of channels in parallel with a distribution of length $R$ :

$$
\begin{aligned}
\overline{G_{\alpha, \beta}^{1,2, A}(\omega) G_{\beta, \alpha}^{2,1, R}(\omega)}= & \frac{1}{\Delta R} \int_{R-\Delta R / 2}^{R+\Delta R / 2} d R \\
& \times G_{\alpha, \beta}^{1,2, A}(R, \omega) G_{\beta, \alpha}^{2,1, R}(R, \omega),
\end{aligned}
$$

with $\Delta R=\lambda_{F}=2 \pi / k_{F}$.

The tunneling limit involves bare Green's functions, which satisfy $\overline{g_{\alpha, \beta}^{1,2, A}(\omega) g_{\beta, \alpha}^{2,1, R}(\omega)}=\overline{g_{\alpha, \beta}^{1,1, A}(\omega) g_{\beta, \alpha}^{1,1, R}(\omega)}$, therefore the crossed conductances for parallel $\left(P_{a}=P_{b}\right)$ and antiparallel $\left(P_{a}=-P_{b}\right)$ spin polarizations are opposite. As a result, the crossed conductance is zero in absence of spin polarization ${ }^{10}$. Similarly, the crossed conductance at a mixed FSN interface (where only one electrode is ferromagnetic) is zero. As shown below, these symmetry properties do not hold anymore for transparent interfaces, where dressing of the quasiparticle propagators occurs in the superconductor.

\section{B. Perturbative expansion in $1 /\left(k_{F} R\right)$}

Now we revisit the perturbative expansion ${ }^{10,12,13}$ used to describe crossed Andreev reflection. We suppose that the contacts $a-\alpha$ and $b-\beta$ can be highly transparent so that multiple Andreev reflections take place locally. However $k_{F} R$ is large so that in a first approximation a single non local process is included in $\hat{G}_{\alpha, \beta}$. This leads to

$$
\hat{G}_{\alpha, \beta}=\hat{M}_{\alpha, \alpha}^{(0)} \hat{g}_{\alpha, \beta} \hat{N}_{\beta, \beta}^{(0)},
$$

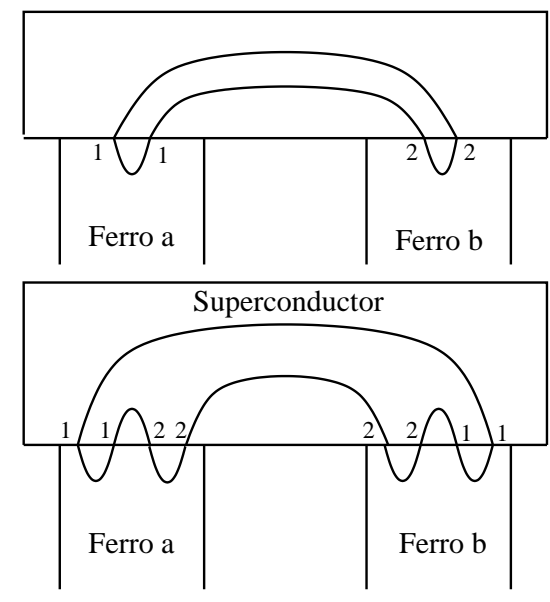

(a)

FIG. 3: (a) A CAR process to order $\tau$ 4. (b) A CAR process to order $\tau^{8}$ involving two local Andreev reflections. The Nambu indices are indicated at each vertex. A similar diagram can be drawn for EC process.

where $\hat{M}_{\alpha, \alpha}^{(0)}\left(\hat{N}_{\beta, \beta}^{(0)}\right)$ describes the dressing by multiple Andreev reflections at the contact $a-\alpha(b-\beta) . \hat{M}_{\alpha, \alpha}^{(0)}$ is determined by the equation $\hat{G}_{\alpha, \alpha}^{(0)}=M_{\alpha, \alpha}^{(0)} \hat{g}_{\alpha, \alpha}$, where $\hat{G}_{\alpha, \alpha}^{(0)}$ is the fully dressed propagator at site $\alpha$ with $t_{b, \beta}=0$. We find easily

$$
\begin{aligned}
\hat{M}_{\alpha, \alpha}^{(0)} & =\left[\hat{I}-\hat{g}_{\alpha, \alpha} \hat{t}_{\alpha, a} \hat{g}_{a, a} \hat{t}_{a, \alpha}\right]^{-1} \\
\hat{N}_{\beta, \beta}^{(0)} & =\left[\hat{I}-\hat{t}_{\beta, b} \hat{g}_{b, b} \hat{t}_{b, \beta} \hat{g}_{\beta, \beta}\right]^{-1} .
\end{aligned}
$$

Generalizing Ref. 10 we evaluate the phase averaging of the following Green's functions:

$$
\begin{aligned}
\overline{\left(g_{\alpha, \beta}^{1,1}\right)^{2}} & =\overline{\left(g_{\alpha, \beta}^{2,2}\right)^{2}}=\overline{\left(g_{\alpha, \beta}^{1,2}\right)^{2}} \\
& =\frac{\pi^{2} \rho_{S}^{2}}{2\left(k_{F} R\right)^{2}} \exp \left(-\frac{2 R}{\xi(\omega)}\right) \frac{\Delta^{2}}{\Delta^{2}-\omega^{2}} \\
\overline{g_{\alpha, \beta}^{1,1} g_{\alpha, \beta}^{2,2}} & =\frac{\pi^{2} \rho_{S}^{2}}{2\left(k_{F} R\right)^{2}} \exp \left(-\frac{2 R}{\xi(\omega)}\right) \frac{2 \omega^{2}-\Delta^{2}}{\Delta^{2}-\omega^{2}} \\
\overline{g_{\alpha, \beta}^{1,1} g_{\alpha, \beta}^{1,2}} & =\frac{g_{\alpha, \beta}^{2,2} g_{\alpha, \beta}^{1,2}}{2\left(k_{F} R\right)^{2}} \exp \left(-\frac{2 R}{\xi(\omega)}\right) \frac{-\omega \Delta}{\Delta^{2}-\omega^{2}}
\end{aligned}
$$

We use Eqs. (25)-(27) and Eq. (22) to evaluate the phase averaged Green's function in the CAR and EC currents given by (18) and (19).

\section{Crossed conductance in a FSF structure}

\section{Normalization of the tunnel amplitudes}

For a NN contact, highly transparent interfaces correspond to $t=t_{0}$, where $t_{0}$ is such that $\pi^{2} t_{0}^{2} \rho_{N}^{2}=1$. The 
NN contact conductance is given by $\mathcal{G}_{N N}=\left(2 e^{2} / h\right) \alpha$, with $^{34}$

$$
\alpha=\frac{4\left(t / t_{0}\right)^{2}}{\left(1+\left(t / t_{0}\right)^{2}\right)^{2}} .
$$

The conductance of a NF contact is given by $\mathcal{G}_{N F}=$ $\left(e^{2} / h\right)\left(\alpha_{\uparrow}+\alpha_{\downarrow}\right)$, with

$$
\begin{aligned}
& \alpha_{\uparrow}=\frac{4 \pi^{2} t^{2} \rho_{N} \rho_{F}(1+P)}{\left[1+\pi^{2} t^{2} \rho_{N} \rho_{F}(1+P)\right]^{2}} \\
& \alpha_{\downarrow}=\frac{4 \pi^{2} t^{2} \rho_{N} \rho_{F}(1-P)}{\left[1+\pi^{2} t^{2} \rho_{N} \rho_{F}(1-P)\right]^{2}}
\end{aligned}
$$

The value $t_{0}$ of $t$ corresponding to a perfect transmission in the spin-up channel is given by

$$
\pi^{2} t_{0}^{2}(P) \rho_{N} \rho_{F}(1+P)=1
$$

The conductance of a SF contact with perfect transmission in the spin-up channel $\left(t=t_{0}(P)\right)$ is given by

$$
\mathcal{G}_{N F}=\frac{e^{2}}{h}\left(1+\frac{4 \frac{1-P}{1+P}}{\left(1+\frac{1-P}{1+P}\right)^{2}}\right),
$$

varying between $e^{2} / h$ for half-metal ferromagnets and $2 e^{2} / h$ in the absence of spin polarization. In the following we normalize the hopping amplitude $t$ to the maximal value $t_{0}(1)$ of $t_{0}(P): t=\tau t_{0}(1)$, with $\tau$ between 0 and 1 .

\section{Linear crossed conductance}

Taking $\omega \ll \Delta$, the linear crossed conductances corresponding to $V_{b}=0$ are given by

$$
\begin{aligned}
\lambda \mathcal{G}_{\mathrm{AP}}= & 4 \frac{e^{2}}{h} \frac{\tau^{4}}{\mathcal{D}^{2}(P)}\left\{-\frac{\tau^{4}\left(1-P^{2}\right)}{\mathcal{D}^{2}(P)}+P^{2}\right\} \\
\lambda \mathcal{G}_{\mathrm{P}}= & 4 \frac{e^{2}}{h} \frac{\tau^{4}}{\mathcal{D}^{2}(P)} \\
& \left\{-\frac{\tau^{4}\left(1-P^{2}\right)\left(1-2 P^{2}\right)}{\mathcal{D}^{2}(P)}-P^{2}\right\},
\end{aligned}
$$

with $\mathcal{D}(P)=1+\tau^{4}\left(1-P^{2}\right) / 4, \lambda=2\left(k_{F} R\right)^{2} \exp \left(2 R / \xi_{0}\right)$ in the ballistic limit, and $\lambda=2\left(k_{F} l_{e}\right)\left(k_{F} R\right) \exp \left(2 R / \xi_{0}\right)$ in the diffusive limit $\frac{16}{}$, where $l_{e}$ is the elastic mean free path and $\xi_{0}$ the coherence length at zero energy. The variations of $\lambda \mathcal{G}_{\mathrm{AP}}(P)$ and $\lambda \mathcal{G}_{\mathrm{P}}(P)$ are shown on Fig. 2 for increasing values of $\tau$. First, one sees that at $P=0$ the crossed conductance is negative, as if a normal metal would replace the superconductor ${ }^{3}$. By contrast, in the tunneling approach, an exact symmetry holds between CAR and EC, so that the crossed conductance is zero for $P_{a}=0$ or $P_{b}=0^{10.12}$. The observed trend is more apparent for transparent contacts, and means that EC processes dominate over CAR. We obtain $\mathcal{G}_{\mathrm{AP}}(P) \simeq-\mathcal{G}_{\mathrm{P}}(P)$ only for small values of $\tau(\tau=0.25$ on Fig. 2-(a)). As $\tau$
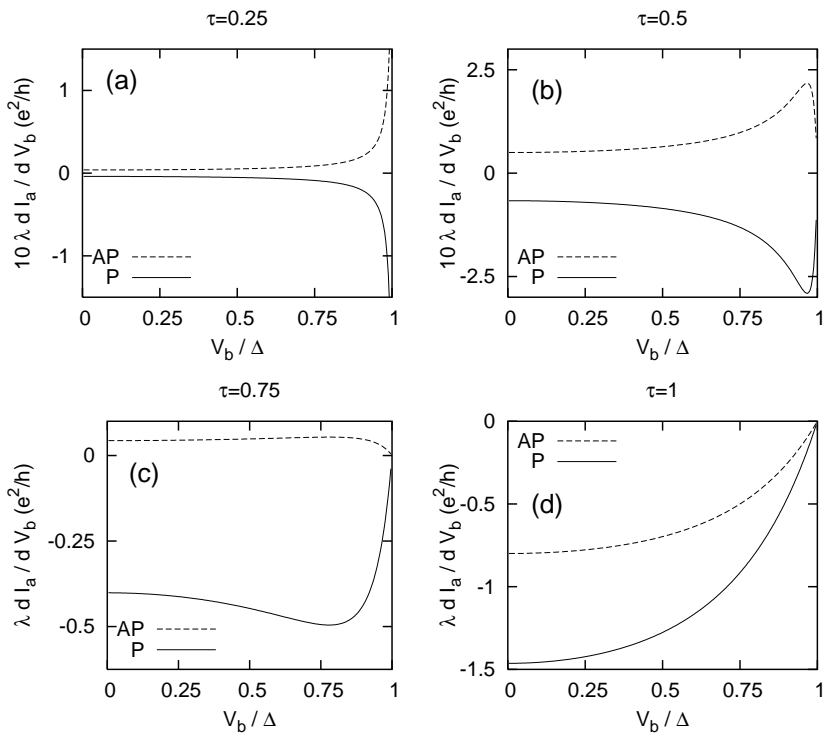

FIG. 4: Variation of the normalized crossed conductance $\lambda\left(V_{b}\right) \mathcal{G}\left(V_{b}\right)=\lambda \partial I_{a} / \partial V_{b}\left(V_{a}=0, V_{b}\right)$ as a function of the normalized voltage $V_{b} / \Delta$, for $P=0.5$ and increasing values of the dimensionless tunnel amplitude $\tau$. The parallel (antiparallel) alignment corresponds to the solid (dashed) line.

is increased $\mathcal{G}_{\mathrm{AP}}(P)$ changes sign as shown on Figs. 2. (b), 2. (c) and 2.(d). For half-metal ferromagnets we have $\mathcal{G}_{\mathrm{AP}}(P)=-\mathcal{G}_{\mathrm{P}}(P)$ for all values of $\tau$, as it can be seen from Eqs. (33) and (34). The key role in the sign changes is played by processes of order $\tau^{8}$ like the one represented on Fig. 3. (b). Due to the opposite signs of $\overline{\left(g_{\alpha, \beta}^{1,1}\right)^{2}}$ and $\overline{g_{\alpha, \beta}^{1,1} g_{\alpha, \beta}^{2,2}}$ for $\omega=0$ (see Eqs. (25] 26)), local Andreev dressing effects tend to decrease $\overline{G_{\alpha, \beta}^{1,2, A} G_{\beta, \alpha}^{2,1, R}}$ and increase $\overline{G_{\alpha, \beta}^{1,1, A} G_{\beta, \alpha}^{1,1, R}}$, therefore CAR is weakened and EC reinforced.

\section{Crossed conductance versus voltage}

The variations of the crossed conductances as a function of the voltage $V_{b}$ applied on electrode "b" are shown on Fig. 4 for $P=0.5$. For small interface transparencies $(\tau=0.25$ and $\tau=0.5)$ the crossed conductances in the parallel and antiparallel alignments are approximately opposite in the entire voltage range. The crossed conductance at $V_{b}=\Delta$ is vanishingly small, both in the parallel and antiparallel alignments and for arbitrary spin polarizations.

For half-metal ferromagnets the crossed conductances take the form

$$
\begin{aligned}
\lambda\left(V_{b}\right) \mathcal{G}_{\mathrm{AP}}\left(V_{b}\right)= & -\lambda\left(V_{b}\right) \mathcal{G}_{\mathrm{P}}\left(V_{b}\right)= \\
& 4 \tau^{4} \frac{\Delta^{2}}{\Delta^{2}-V_{b}^{2}}\left(1+\tau^{4} \frac{V_{b}^{2}}{\Delta^{2}-V_{b}^{2}}\right)^{-2},
\end{aligned}
$$

where $\lambda\left(V_{b}\right)$ has the same expression as $\lambda$ except that 
$\xi_{0}$ is replaced by the coherence length at a finite energy $\xi_{0} \Delta / \sqrt{\Delta^{2}-V_{b}^{2}}$. Eq. (35) is approximately equal to $\left(8 / \tau^{4}\right)\left(1-V_{b} / \Delta\right)$ if $\Delta-\omega \ll \tau^{4} \Delta$, and approximately equal to $4 \tau^{4} \Delta^{2} /\left(\Delta^{2}-V_{b}^{2}\right)$ if $\Delta-V_{b} \gg \tau^{4} \Delta$. For $\tau$ small there is thus a maximum in the crossed conductance $\frac{12}{2}$ at a voltage $V_{b} \simeq \Delta\left(1-\tau^{4} / 2\right)$. This argument for $P=1$ is compatible with the behavior of the crossed conductances

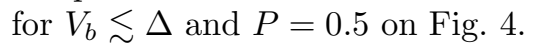

\section{Crossed conductance of a FSN or NSF junction} tion

The linear crossed conductance of a FSN (NSF) junc-

$$
\lambda \mathcal{G}=-\frac{e^{2}}{h} \frac{8 \tau^{8}\left(1-P^{2}\right)}{\left(1+\tau^{4}\left(1-P^{2}\right) / 4\right)\left(1+\tau^{4} / 4\right)}
$$

is proportional to $\tau^{8}$ and thus very small for tunnel interfaces. However it can take measurable values for highly transparent interfaces and with a weakly polarized ferromagnet or even a normal metal.

\section{Interpretation of the dressing by local processes}

One of the terms contributing to $G_{\alpha, \beta}^{1,1, A} G_{\beta, \alpha}^{1,1, A}$ in the parallel alignment is

$$
M_{\alpha, \alpha}^{(0), 1,2} g_{\alpha, \beta}^{2,1} N_{\beta, \beta}^{(0), 1,1} M_{\beta, \beta}^{(0), 1,2} g_{\beta, \alpha}^{2,1} N_{\alpha, \alpha}^{(0), 1,1},
$$

from what we deduce one of the terms of order $t_{a}^{4} t_{b}^{4}$ contributing $t_{a}^{2} t_{b}^{2} \rho_{a, \uparrow} \rho_{b, \uparrow} G_{\alpha, \beta}^{1,1, A} G_{\beta, \alpha}^{1,1, A}$ :

$$
\begin{aligned}
& t_{\alpha, a}^{1,1} \rho_{a, a}^{1,1} t_{a, \alpha}^{1,1} g_{\alpha, \alpha}^{1,2} t_{\alpha, a}^{2,2} g_{a, a}^{2,2, A} t_{a, \alpha}^{2,2} g_{\alpha, \beta}^{2,1} \\
& \times t_{\beta, b}^{1,1} \rho_{b, b}^{1,1} t_{b, \beta}^{1,1} g_{\beta, \beta}^{1,2} t_{\beta, b}^{2,2} g_{b, b}^{2,2, R} t_{b, \beta}^{2,2} g_{\beta, \alpha}^{2,1}
\end{aligned}
$$

This process corresponds to a diagram in which a spin-up electron from electrode "a" is locally Andreev reflected as a spin-down hole that makes an excursion in electrode "a", is converted in a spin-up electron in electrode "b" through a CAR. The spin-up electron in electrode "b" undergoes a local Andreev reflection, is converted in a spin-down hole that is transformed in a spin-up electron by a CAR. This process is not possible for half-metal ferromagnets, in which case the linear conductance is equal to the bare conductance, proportional to $\tau^{4}$.

\section{INFINITE PLANAR INTERFACES WITH A BULK SUPERCONDUCTOR}

Now we consider infinite planar 2D interfaces connecting a bulk 3D superconductor to bulk 3D ferromagnets. Within this approach we do not have to impose "by hand" phase averaging as we did in the previous section (see Eqs. (25)-(27)). We use the mixed Green's functions introduced in section IIID The evaluation of the integrals
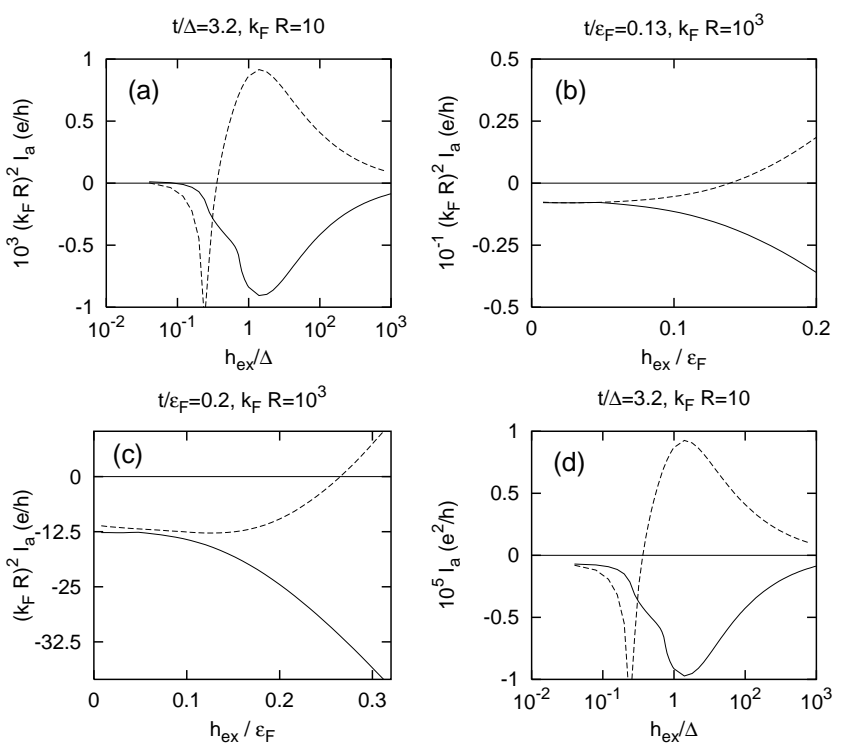

FIG. 5: Variation of the total current $I_{a}$ through electrode "a" evaluated with $e V_{a}=0$ and $e V_{b}=\Delta / 2$ as a function of the exchange field, with $\Delta / \epsilon_{F}=4 \times 10^{-3}$. The parallel (antiparallel) alignment corresponds to the solid (dashed) line. (a), (b) and (c) correspond to a full Green's function calculation with CAR and EC to all orders. (d) corresponds to the approximation given by Eq. (22). We use $\eta_{S} / \Delta=10^{-1}$ and $\eta_{F} / \epsilon_{F}=0.4$ but obtained similar results for $\eta_{F} / \Delta=10^{-1}$.

in the expression of the CAR and EC currents is briefly described in Appendix A A realistic model would involve an estimate of the inverse proximity effect, i.e. how the superconducting gap depends on the relative spin orientation of the ferromagnetic electrodes $25,26,27,28,29$. Here we consider the infinite planar limit just as a test of the approach in the preceding section and do not impose selfconsistency on the superconducting gap.

We calculate the crossed current through electrode "a" with a finite voltage $V_{b}$ on electrode "b", and with $V_{a}=0$ and suppose that the distance between the ferromagnets is much larger than the Fermi wave-length. The variations of the current evaluated at $V_{b}=\Delta / 2$ as a function of $h_{\mathrm{ex}} / \Delta$ is shown on Fig. 罒 for different values of the distance $R$ between the contacts. For small values of $t / \Delta$ we obtain one Andreev bound state for weak ferromagnets for which $h_{\mathrm{ex}}$ and $\Delta$ have the same order of magnitude (see Fig. 5.(a)). Andreev bound states for weak ferromagnets were already discussed elsewhere ${ }^{29}$. For larger values of $t / \Delta$ and larger values of $R / a_{0}$ (see Fig. 5 -(b)) we obtain $I_{\mathrm{AP}} \simeq I_{\mathrm{P}}$ up to relatively large values of $h_{\mathrm{ex}} / \Delta$ and $I_{\mathrm{AP}} \simeq-I_{\mathrm{P}}$ for the largest values of $h_{\mathrm{ex}} / \Delta$. The overall variation on Fig. 5. (a), Fig. 5 -(b) and Fig. 5 . (c) looks like Fig. 2.(a), Fig. 2.(b) and Fig. 2.(c). Fig. 5 .(d) corresponds to the same parameters as Fig. 5. (a) except that we used the approximation (22) of a single non local process on Fig. 5 . (d) whereas Fig. 5 . (a) was obtained without approximation. The two variations are almost identical, except for the regime $h_{\mathrm{ex}} \ll \Delta$. The agree- 
ment is even better for larger values of $R$ (not shown on Fig. [5). This illustrates the validity of the approximation (22) in which we keep a single CAR.

\section{CONCLUSIONS}

To conclude we have proposed a model that may constitute an explanation to the signs of the crossed currents in the recent experiment by Beckmann et al. ${ }^{21}$. In the experiment, a current is injected through electrode $b$, and a voltage $V_{a}$ is measured. Assuming that the local conductances $\mathcal{G}_{a a}, \mathcal{G}_{b b}$ are much larger than $\mathcal{G}_{a b}, \mathcal{G}_{b a}$, one simply gets the non-local resistance $\mathcal{R}_{a b}^{\mathrm{P}, \mathrm{AP}} \sim-\mathcal{G}_{a b}^{\mathrm{P}, \mathrm{AP}} / \mathcal{G}_{a a} \mathcal{G}_{b b}$. For small interface transparencies we recover the results of perturbation theory to order $t_{a}^{2} t_{b}^{2}$ : the crossed conductance in the antiparallel alignment $\mathcal{G}_{a b}^{\mathrm{AP}}$ is opposite to the crossed conductance $\mathcal{G}_{a b}^{\mathrm{P}}$ in the parallel alignment, and is zero if $P_{a}=0$ or $P_{b}=0$. For larger interface transparencies, the propagators of crossed Andreev reflection and elastic cotunneling are dressed by local Andreev reflections. As a result $\mathcal{G}_{a b}^{\mathrm{AP}}$ can become negative as the interface transparencies are increased, whereas $\mathcal{G}_{a b}^{\mathrm{P}}$ is always negative. We also carried out simulations of infinite planar interfaces and found the same qualitative behavior without imposing phase averaging by hand and without using the approximation in which a single non local process is retained.

\section{Acknowledgments}

One of the authors (D.F.) acknowledges stimulating discussions with G. Deutscher and F. Sols.

\section{APPENDIX A: EVALUATION OF INTEGRALS FOR INFINITE PLANAR INTERFACES}

In this Appendix we give necessary technical details regarding changes of variable for evaluating the current with infinite planar interfaces. The " $11 "$ component of the CAR current takes the form

$$
\begin{aligned}
& I_{1,1}^{\mathrm{CAR}}=4 \pi^{3} t_{a}^{2} t_{b}^{2} \frac{e}{h}\left(\frac{2 m a_{0} 2}{\hbar 2}\right) 2 \int_{-e V_{b}}^{e V_{a}} d \omega \int_{-D}^{D} d \xi_{\|}(\mathrm{A} 1) \\
& \rho_{1,1}^{a, a}\left(\omega-e V_{a}, \xi_{\|}\right) \rho_{2,2}^{b, b}\left(\omega-e V_{b}, \xi_{\|}\right)\left|G_{1,2}^{A, \alpha}\left(\omega, \xi_{\|}\right)\right|^{2}
\end{aligned}
$$

and the "11" component of the EC current takes the form

$$
\begin{aligned}
& I_{1,1}^{\mathrm{EC}}=4 \pi^{3} t_{a}^{2} t_{b}^{2} \frac{e}{h}\left(\frac{2 m a_{0}^{2}}{\hbar^{2}}\right)^{2} \int_{e V_{b}}^{e V_{a}} d \omega \int_{-D}^{D} d \xi_{\|} \\
& \rho_{1,1}^{a, a}\left(\omega-e V_{a}, \xi_{\|}\right) \rho_{1,1}^{b, b}\left(\omega-e V_{b}, \xi_{\|}\right)\left|G_{1,1}^{A, \alpha, \beta}\left(\omega, \xi_{\|}\right)\right|^{2}
\end{aligned}
$$

Similar expressions are obtained for the " 22 " currents and for the currents in the spin-down sector.

The density of state $\rho_{1,1}^{a, a}\left(\omega-e V_{a}, \xi_{\|}\right)$and $\rho_{1,1}^{b, b}(\omega-$ $\left.e V_{b}, \xi_{\|}\right)$deduced from Eq. 14 contain a square root singularity. For instance

$$
\rho_{1,1}^{a, a}\left(\omega-e V_{a}, \xi_{\|}\right)=\frac{\sqrt{2 m a_{0}^{2}}}{\hbar} \frac{\theta\left(\omega-e V_{a}-\xi_{\|}+h_{a}\right)}{\sqrt{\omega-e V_{a}-\xi_{\|}+h_{a}}}
$$

After a change of variable $I_{1,1}^{\mathrm{CAR}}$ takes the form

$$
\begin{aligned}
I_{1,1}^{\mathrm{CAR}}= & 8 \pi^{3} t_{a}^{2} t_{b}^{2} \frac{e}{h}\left(\frac{2 m a_{0}^{2}}{\hbar^{2}}\right) \int_{x_{\min }}^{x_{\max }} d x \int_{0}^{u_{\max }} \\
& \frac{d u}{\sqrt{2 x+u^{2}}}\left|G_{1,2}^{\alpha, \beta}\left(\omega(x), \xi_{\|}\right)\right|^{2}
\end{aligned}
$$

where explicit expressions of $x(\omega), x_{\min }, x_{\max }$ and $u_{\max }$ can be obtained in each case. For instance if $-e V_{b}<e V_{a}$ we have $x(\omega)=-\omega+e\left(V_{a}+V_{b}\right) / 2-\left(h_{a}+h_{b}\right) / 2$ and $u=\sqrt{\omega-e V_{a}-\xi_{\|}+h_{a}}$. The integral (A4) is then evaluated by making the changes of variable $v^{2}=2 x$ and $(u, v)=\rho(\cos \theta, \sin \theta)$, therefore absorbing the square root singularity in a change of variable.
1 A.F. Andreev, Zh. Eksp. Teor. Fiz. 46, 1823 (1964) [Sov. Phys. JETP 19, 1228 (1964)].

2 P. G. De Gennes and D. Saint-James, Phys. Lett. 4, 151 (1963); D. Saint-James, J. de Physique (Paris) 25, 899 (1964).

3 N. K. Allsopp, V. C. Hui, C. J. Lambert and S. J. Robinson, J. Phys.: Condens. Matter 6, 10475 (1994).

4 C.J. Lambert and R. Raimondi, J. Phys.: Condens. Matter 10, 901 (1998).

${ }^{5}$ F.J. Jedema, B.J. van Wees, B.H. Hoving, A.T. Filip and T.M. Klapwijk, Phys. Rev. B 60, 16549 (1999); B. J. van Wees et al. , "Mesoscopic Electron Transport" ed. L. Sohn, L. Kouwenhoven and G. Schön, NATO ASI Series E: vol 345 (1996).
${ }^{6}$ M.J.M. de Jong and C.W.J. Beenakker, Phys. Rev. Lett. 74, 1657 (1995).

7 R.J. Soulen et al., Science 282, 85 (1998).

8 S.K. Upadhyay et al., Phys. Rev. Lett. 81, 3247 (1998).

${ }^{9}$ G. Deutscher and D. Feinberg, App. Phys. Lett. 76, 487 (2000).

10 G. Falci, D. Feinberg, and F.W.J. Hekking, Europhys. Lett. 54, 255 (2001).

11 R. Mélin, J. Phys.: Condens. Matter 13, 6445 (2001);

12 R. Mélin and D. Feinberg, Eur. Phys. J. B 26, 101 (2002).

13 R. Mélin and S. Peysson, Rev. B 68, 174515 (2003)

14 N.M. Chtchelkatchev, I.S. Burmistrov, Phys. Rev. B 68, 140501 (2003).

15 R. Mélin, H. Jirari and S. Peysson, J. Phys.: Condens. 
Matter 15, 5591 (2003).

16 D. Feinberg, Eur. Phys. J. B 36, 419 (2003).

17 D. Sanchez, R. Lopez, P. Samuelsson and M. Buttiker, Phys. Rev. B 68, 214501 (2003).

18 F. Taddei and R. Fazio, Phys. Rev. B 65, 134522 (2002).

19 G. Bignon, M. Houzet, F. Pistolesi, and F. W. J. Hekking, Europhys. Lett. 67, 110 (2004).

20 C.J. Lambert, J. Koltai, and J. Cserti, in Towards the controllable quantum states (Mesoscopic superconductivity and spintronics, p. 119, Eds H. Takayanagi and J. Nitta, World Scientific (2003).

21 D. Beckmann, H.B. Weber and H.v; Löhneysen, cond-mat/0404360

22 T. Yamashita, S. Takahashi and S. Maekawa, Phys. Rev. B 68174504 (2003).

${ }^{23}$ G. E. Blonder, M. Tinkham and T. M. Klapwijk, Phys. Rev. B25, 4515 (1982)

24 D.V. Averin and Yu.V. Nazarov, in "Single Charge Tunneling", Chap. 6, ed. by H. Grabert and M.H. Devoret, Plenum Press, New York 1992.

25 V. Apinyan and R. Mélin, Eur. Phys. J. B 25, 373 (2002).

${ }^{26}$ H. Jirari, R. Mélin and N. Stefanakis, Eur. Phys. J. B 31,
125 (2003).

27 A. Buzdin and M. Daumens, Europhys. Lett. 64, 510 (2003).

28 R. Mélin and D. Feinberg, Europhys. Lett. 65, 96 (2004).

29 R. Mélin, Eur. Phys. J. B 39, 249 (2004).

${ }^{30}$ G. E. Blonder, Ph. D. thesis, Harvard University, 1982 (unpublished).

31 M. Tinkham, Introduction to superconductivity, Second Edition, Mc Graw-Hill (1996).

32 A.A. Abrikosov, L.P. Gorkov, and I.E. Dzyaloshinski, Methods of quantum field theory in statistical physics, Dover (1975).

33 S.B. Kaplan, C. C. Chi, D. N. Langenberg, J. J. Chang, S. Jafarey and D. J. Scalapino, Phys. Rev. B 14, 4854 (1976).

34 J.C. Cuevas, A. Martín-Rodero and A. Levy Yeyati, Phys. Rev. B 54, 7366 (1996).

35 R. Mélin, cond-mat/0406275

36 C. Caroli, R. Combescot, P. Nozières and D. Saint-James, J. Phys. C: Solid St. Phys. 4, 916 (1971); ibid. 5, 21 (1972).

37 A mistake is present in Ref. 12, in the last matrix of expression (12). 